Homology, Homotopy and Applications, vol.10(2), 2008, pp.139-148

\title{
INVOLUTIONS ON 3-MANIFOLDS AND SELF-DUAL, BINARY CODES
}

\author{
MATTHIAS KRECK AND VOLKER PUPPE
}

\author{
(communicated by Gunnar Carlsson)
}

\begin{abstract}
We study a correspondence between orientation-reversing involutions on compact 3-manifolds with only isolated fixed points and self-dual, binary codes. We show in particular that every such code can be obtained from such an involution. We further relate doubly even codes to $\mathrm{Pin}^{-}$-structures and Spinmanifolds.
\end{abstract}

\section{Introduction}

A self-dual, binary code $C$ of length $k$ is a linear subspace of the $k$-dimensional vector space $(\mathbb{Z} / 2)^{k}$ over the field $\mathbb{Z} / 2$, which coincides with its orthogonal complement with respect to the standard "Euclidean" form $\langle x, y\rangle:=\sum_{i} x_{i} y_{i} \in \mathbb{Z} /{ }_{2}$, for $x=\left(x_{1}, \ldots, x_{k}\right), y=\left(y_{1}, \ldots, y_{k}\right) \in(\mathbb{Z} / 2)^{k}$. The weight, $w t(x)$, of a code word $x \in C$ is defined as the number of non-zero coordinates in $x=\left(x_{1}, \ldots, x_{k}\right)$. It is immediate that all code words in a self-dual, binary code $C$ have even weights, and it is also easy to see, that the vector $\Delta:=(1, \ldots, 1)$ always belongs to $C$.

Self-dual, binary codes play an important role in coding theory and its applications, and have been studied extensively; see [5] for a comprehensive survey and literature. They have interesting relations to many different mathematical areas. A connection to involutions on 3-manifolds was made in [4]. It is shown there that an involution $\tau: M \rightarrow M$ on a 3-dimensional, closed manifold $M$ with the "maximal number" of isolated fixed points (i.e. with only isolated fixed points, such that the number of fixed points $k:=\left|M^{\tau}\right|$ equals $\left.\operatorname{dim}_{\mathbb{Z} / 2}\left(\oplus_{i} H^{i}(M ; \mathbb{Z} / 2)\right)\right)$ determines a self-dual, binary code of length $k$. In turn this code determines the cohomology algebra $H^{*}(M ; \mathbb{Z} / 2)$ and the equivariant cohomology $H_{G}^{*}(M \mathbb{Z} / 2)$, where the action of $G:=\mathbb{Z} / 2$ is given by the involution. In fact, the code corresponds to the inclusion $H_{G}^{*}(M \mathbb{Z} / 2) \longrightarrow H_{G}^{*}\left(M^{G} ; \mathbb{Z} / 2\right) \cong(\mathbb{Z} / 2[t])^{k}$, and an equivalence of codes, given by permuting the coordinates corresponds to an equivalence of inclusions, given by an automorphism of the algebra $(\mathbb{Z} / 2[t])^{k}$.

At the moment we have no constructions of 3-manifolds with involution having finitely many fixed points leading to new codes, but we hope that our work motivates the search for such examples. In turn self-dual and in particular doubly even codes,

Received June 3, 2007, revised April 15, 2008, June 5, 2008; published on September 4, 2008. 2000 Mathematics Subject Classification: 57M60, 94B05, 57M50, 57R91.

Key words and phrases: involution, 3-manifold, code, cohomology.

This article is available at http://intlpress.com/HHA/v10/n2/a7

Copyright (C) 2008, International Press. Permission to copy for private use granted. 
i.e. self-dual, binary codes for which all code words have weights zero mod 4 are very rare, indicating that 3 -manifolds with a symmetry given by such an involution are hard to find. The fact that self-dual codes are rare is indicated by their interesting relation to positive definite unimodular intergral bilinear forms, which are very hard to find and by no means understood. Namely if $C \subset(\mathbb{Z} / 2)^{k}$ is a self-dual code, then $L:=\left\{x / \sqrt{2} \in \mathbb{R}^{k} \mid x(\bmod 2) \in C\right\}$ is a unimodular lattice and an even lattice if $C$ is doubly even (cf. [5]).

In Section 2 we generalize results from [4] by considering involutions on 3-manifolds which have a finite number of fixed points which need not be maximal. The code corresponding to such an involution is described in two ways: firstly using equivariant cohomology as in [4], secondly using the ordinary homology (with $\mathbb{Z} / 2$ coefficients) of the complement (of a neighbourhood) of the fixed points in the orbit space.

In Section 3 we show that every binary, self-dual code can be obtained from an involution on a 3-manifold with a finite number of fixed points; in fact, using surgery we get that the manifold can be chosen so that the number of fixed points is maximal. If the latter holds, then the code determines the (equivariant) cohomology algebra of the manifold, as already mentioned above.

In Section 4 we relate doubly even codes to Spin-manifolds. We define the concept of a Spin-involution and show that Spin-involutions give doubly even codes. Finally we show that each doubly even code comes from a 3-manifold with Spin-involution.

\section{Self-dual codes from involutions on 3-manifolds}

Let $\tau: M \rightarrow M$ be an involution on a closed 3-manifold $M$ with finitely many fixed points $x_{1}, \ldots, x_{k}$. By Smith theory, $k \leqslant \operatorname{dim}_{\mathbb{Z} / 2}\left(\oplus_{i} H^{i}(M ; \mathbb{Z} / 2)\right)$; we say that the number of fixed points is maximal, if equality holds. The Localization Theorem for equivariant cohomology (cf. e.g. [1]) in this context reads as follows (here and in the following we always take coefficients in $\mathbb{Z} / 2$ ):

The map

$$
H_{G}^{*}(M) \stackrel{i_{G}^{*}}{\longrightarrow} H_{G}^{*}\left(M^{G}\right) \cong(\mathbb{Z} / 2[t])^{k}
$$

induced by the inclusion $M^{G} \stackrel{i}{\longrightarrow} M$ becomes an isomorphism after inverting the powers of $t \in \mathbb{Z} /{ }_{2}[t]=H^{*}(B G)$. (Here $H_{G}^{*}\left(M^{G}\right) \cong\left(\mathbb{Z} /{ }_{2}[t]\right)^{k}$ is the canonical isomorphism of $\mathbb{Z} /{ }_{2}[t]$-algebras, given by the inclusion of the single fixed points into $M^{G}$.)

By the Localization Theorem, the kernel of $i_{G}^{*}$ is the torsion submodule $T \subset H_{G}^{*}(M)$, and $i_{G}^{*}$ is injective if and only if $H_{G}^{*}(M)$ is a free $\mathbb{Z} /{ }_{2}[t]$-module. We show that, as in the "maximal number of fixed points" case (see [4]), the inclusion

$H_{G}^{*}(M) / T \stackrel{\bar{i}_{G}^{*}}{\longrightarrow}\left(\mathbb{Z} /{ }_{2}[t]\right)^{k}$ determines a self-dual code $C(M, \tau)$, and in turn $\bar{i}_{G}^{*}$ is determined by this code.

In more detail: Let $C(M, \tau)$ be the image of $\oplus_{i=0}^{1} H_{G}^{i}(M)$ in $(\mathbb{Z} / 2)^{k}$ under the evaluation map, putting $t=1$ (cf. [4]). This is the same as $\Im\left(i_{G}^{1}\right)$, the image of $H_{G}^{1}(M)$ in $H_{G}^{1}\left(M^{G}\right) \cong(\mathbb{Z} / 2)^{k}$. We claim that $C(M, \tau) \subset(\mathbb{Z} / 2)^{k}$ is self-dual, binary code. This is shown in [4] for the "maximal case" (i.e. $T=0$ ), and the only additional information we need here, in order to apply the same arguments as in the "maximal case", is that $\left(H_{G}^{*}(M) / T\right) \otimes_{\mathbb{Z} / 2[t]} \mathbb{Z} /{ }_{2}$ fulfills Poincaré duality with respect to the induced product. 
This can be seen as follows (cf. [1, Exercise (1.15)]):

Let $\rho: H_{G}^{*}(M) \rightarrow H^{*}(M)$ be the restriction to the fibre in the Borel construction $M \rightarrow M \times_{G} E G \rightarrow B G$. The map $\rho$ fits into the long exact Gysin sequence of the covering $M \simeq M \times E G \rightarrow M \times_{G} E G$; i.e.,

$$
\cdots \rightarrow H_{G}^{i-1}(M) \stackrel{\cup t}{\rightarrow} H_{G}^{i}(M) \stackrel{\rho}{\rightarrow} H^{i}(M) \rightarrow H_{G}^{i}(M) \stackrel{\cup t}{\rightarrow} H_{G}^{i+1}(M) \rightarrow \cdots
$$

is exact and hence so is

$$
0 \rightarrow H_{G}^{*}(M) \otimes_{\mathbb{Z} / 2[t]} \mathbb{Z} / 2 \rightarrow H^{*}(M) \rightarrow \operatorname{Tor}^{\mathbb{Z} / 2[t]}\left(H_{G}^{*}(M), \mathbb{Z} / 2\right) \rightarrow 0 .
$$

Splitting $H_{G}^{*}(M) \cong T \oplus F$ into a direct sum of the torsion submodule $T$ and a free complement $F$, one sees that $\operatorname{dim}_{\mathbb{Z} / 2}\left(T \otimes_{\mathbb{Z} / 2[t]} \mathbb{Z} / 2\right)=\operatorname{dim}_{\mathbb{Z} / 2} \operatorname{Tor}^{\mathbb{Z} / 2[t]}\left(H_{G}^{*}(M), Z /{ }_{2}\right)$. We claim that with respect to the Poincaré duality pairing in $H^{*}(M)$, the orthogonal complement of $\rho\left(H_{G}^{*}(M)\right)$ is $\rho(T)$. Because of the dimension equality above it suffices to show that $\langle x, y\rangle=\sigma(x \cup y)=0$ for $x \in \rho\left(H_{G}^{*}(M)\right)$ and $y \in \rho(T)$, where $\sigma: H^{*}(M) \rightarrow \mathbb{Z} / 2$ is the orientation of $M$. If $\tilde{x}$ and $\tilde{y}$ are liftings of $x$ and $y$ with respect to $\rho$, then $\tilde{x} \cup \tilde{y} \in T$. Therefore $\tilde{x} \cup \tilde{y}$ is mapped to zero under the equivariant orientation $\tilde{\sigma}: H_{G}^{*}(M) \rightarrow \mathbb{Z} / 2[t]$ (cf. e.g. [1, Chap. 5]). Hence $\tilde{\sigma}(\tilde{x} \cup \tilde{y})=0$ and thus $\sigma(x \cup y)=0$. We get that the graded algebra $\rho\left(H_{G}^{*}(M)\right) / \rho(T) \cong\left(H_{G}^{*}(M) / T\right) \otimes_{\mathbb{Z} / 2_{2}[t]}$ $\mathbb{Z} / 2$ is a subquotient of $H^{*}(M)$, which fulfills Poincaré duality, and $H_{G}^{*}(M) / T$ as a $\mathbb{Z} / 2[t]$-module is isomorphic to $\left(\rho\left(H_{G}^{*}(M)\right) / \rho(T)\right) \otimes_{\mathbb{Z} / 2} \mathbb{Z} / 2[t]$.

We therefore have, analogous to the case $T=0$ (cf. [4]), that

$$
H_{G}^{*}(M) / T \stackrel{\bar{i}_{G}^{*}}{\longrightarrow}(\mathbb{Z} / 2[t])^{k}
$$

determines a self-dual, binary code; namely, $C(M, \tau)=\Im\left(\bar{i}_{G}^{1}\right)=\Im\left(i_{G}^{1}\right)$ and, in turn, is determined by this code. Note, though, that we only get the quotient algebra $H_{G}^{*}(M) / T$ (and $\left.\rho\left(H_{G}^{*}(M)\right) / \rho(T) \cong\left(H_{G}^{*}(M) / T\right) \otimes_{\mathbb{Z} / 2[t]} \mathbb{Z} / 2\right)$ from the code, which means that in case $T \neq 0$ the algebras $H_{G}^{*}(M)$ and $H^{*}(M)$ are not completely determined by the code. Hence the case $T=0$ (i.e. the maximal number of fixed points) is of particular interest, since then the cohomology algebra $H^{*}(M)$ and the equivariant cohomology algebra $H_{G}^{*}(M)$ are completely given by the code $C(M, \tau)$.

In view of the construction below, we describe the code coming from an involution $\tau: M \rightarrow M$ on a closed 3-manifold $M$ with isolated fixed points $x_{1}, \ldots, x_{k}$ in a second way.

Let $W:=\left(M \backslash+{ }_{k} \stackrel{\circ}{D^{3}}\right) / \tau$, where $D_{i}^{3}$ are equivariant discs around $x_{i}$. We consider the Mayer-Vietoris sequence for $M=\left(M \backslash+{ }_{k} \stackrel{\circ}{D^{3}}\right) \cup\left(+{ }_{k} D^{3}\right)$ :

$$
\cdots \rightarrow H_{G}^{0}\left(+{ }_{k} S_{1}^{2}\right) \rightarrow H_{G}^{1}(M) \rightarrow H_{G}^{1}\left(M \backslash+{ }_{k} \stackrel{\circ}{D^{3}}\right) \oplus H_{G}^{1}\left(+{ }_{k} D^{3}\right) \rightarrow H_{G}^{1}\left(+{ }_{k} S^{2}\right) \rightarrow \cdots .
$$

Since the equivariant cohomology of a free $G$-space is the non-equivariant cohomology of the orbit space, one gets the exact sequence

$$
\cdots \rightarrow H^{0}\left(+_{k} \mathbb{R} P^{2}\right) \rightarrow H_{G}^{1}(M) \rightarrow H^{1}(W) \oplus\left(H_{G}^{1}\left(+_{k} D^{3}\right) \rightarrow H^{1}\left(+_{k} \mathbb{R} P^{2}\right) \rightarrow \cdots .\right.
$$

It is easy to see that the map $\left(H_{G}^{1}\left(+{ }_{k} D^{3}\right) \rightarrow H^{1}\left({ }_{k} \mathbb{R} P^{2}\right)\right.$ is an isomorphism. (For one disk $D^{3}$, one has that $S^{2} \stackrel{(i d, i)}{\longrightarrow} S^{2} \times S^{\infty} \stackrel{(j, i d)}{\longrightarrow} D^{3} \times S^{\infty} \stackrel{p}{\rightarrow} S^{\infty}$ is the canonical inclusion, and hence $H_{G}^{1}\left(D^{3}\right) \cong H^{1}\left(\mathbb{R} P^{\infty}\right) \rightarrow H^{1}\left(\mathbb{R} P^{2}\right)=H_{G}^{1}\left(S^{2}\right)$. It therefore follows from 
the Mayer-Vietoris sequence that $C(M, \tau):=\Im\left(H_{G}^{1}(M) \rightarrow H_{G}^{1}\left(M^{G}\right)\right)$ can be identified with $\Im\left(H^{1}(W) \rightarrow H^{1}\left(+_{k} \mathbb{R} P^{2}\right)\right)$. Dually to taking $\Im\left(H^{1}(W) \rightarrow H^{1}\left(+_{k} \mathbb{R} P^{2}\right)\right)$, we can take $\operatorname{Ker}\left(H_{1}\left(+{ }_{k} \mathbb{R} P^{2}\right) \rightarrow H_{1}(W)\right)$. The cup product in cohomology corresponds to the intersection form in homology. Since the kernel of the map on the middle homology of the boundary of a compact manifold to the interior is a self-annihilating subspace (with respect to the intersection form) of half rank, and the intersection form on $H_{1}\left(+{ }_{k} \mathbb{R} P^{2}\right)=(\mathbb{Z} / 2)^{k}$ is the standard "Euclidean" form, one gets in another way that the code is self-dual.

We summarize the above considerations as

Theorem 2.1. Every involution $\tau$ with only isolated fixed points on a compact 3-manifold $M$ determines a self-dual, binary code $C(M, \tau)$.

\section{All self-dual codes come from 3-manifolds}

Proposition 3.1. Every self-dual, binary code can be obtained from an involution on an orientable 3-manifold.

Proof. Let $k=2 r$ and let $C \subset(\mathbb{Z} / 2)^{k}$ be a self-dual, binary code. We choose a map $f:+_{k} \mathbb{R P}^{2} \rightarrow\left(\mathbb{R P}^{\infty}\right)^{r}$ such that the sequence (with $\mathbb{Z}_{2}$ coefficients)

$$
0 \rightarrow C \rightarrow H_{1}\left(+{ }_{k} \mathbb{R} \mathbb{P}^{2}\right) \stackrel{f_{*}}{\rightarrow} H_{1}\left(\left(\mathbb{R} \mathbb{P}^{\infty}\right)^{r}\right) \rightarrow 0
$$

is exact.

Next we note that the first Stiefel-Whitney class $w_{1}\left({ }_{k} \mathbb{R} \mathbb{P}^{2}\right)$ is in the image $f^{*}$. The reason is that the diagonal element $\Delta$ is in the code $\left(\Delta\right.$ is dual to $\left.w_{1}\left(+_{k} \mathbb{R P}^{2}\right)\right)$ and so $\left\langle w_{1}\left({ }_{k} \mathbb{R P}^{2}\right), x\right\rangle=\langle\Delta, x\rangle=0$ for all $x \in C$. This implies that there is a real line bundle $L$ over $\left(\mathbb{R P}^{\infty}\right)^{r}$ pulling back to the non-trivial line bundle over each copy of $\mathbb{R P}^{2}$. Thus $\left(+_{k} \mathbb{R P}^{2}, f\right)$ is an orientable singular manifold, where the orientation is twisted by the line bundle $L$. This means that the bundle $\nu\left({ }_{k} \mathbb{R P}^{2}\right)-f^{*}(L)$ is orientable.

After choosing an orientation the pair, $\left(+_{k} \mathbb{R} P^{2}, f\right)$ represents an element in the bordism group $\left(\Omega_{2}\left(\left(\mathbb{R} P^{\infty}\right)^{r} ; L\right)\right.$ of singular manifold with orientation (twisted by $L$ ). We claim that this element is trivial.

The Atiyah-Hirzebruch spectral sequence implies that

$$
\begin{gathered}
\Omega_{2}\left(\left(\mathbb{R P}^{\infty}\right)^{r} ; L\right) \stackrel{\cong}{\longrightarrow} H_{2}\left(\left(\mathbb{R P}^{\infty}\right)^{r} ; \mathbb{Z}_{t}\right), \\
{[F, h] \longrightarrow h_{*}([F]) .}
\end{gathered}
$$

Here, $\mathbb{Z}_{t}$ stands for twisted homology, where the coefficient system is given by the representation

$$
\pi_{1}\left(\left(\mathbb{R} \mathbb{P}^{\infty}\right)^{r} \rightarrow \pi_{1}\left(\mathbb{R} \mathbb{P}^{\infty}\right) \stackrel{\cong}{\longrightarrow}( \pm 1)=\operatorname{Aut}(\mathbb{Z}),\right.
$$

and the map is induced by the classifying map of $L$. We note that

$$
H_{2}\left(\left(\mathbb{R} \mathbb{P}^{\infty}\right)^{r} ; \mathbb{Z}_{t}\right) \rightarrow H_{2}\left(\left(\mathbb{R} \mathbb{P}^{\infty}\right)^{r} ; \mathbb{Z} / 2\right)
$$

is injective. The reason is that $H_{2}\left(\left(\mathbb{R} \mathbb{P}^{\infty}\right)^{r} ; \mathbb{Z}_{t}\right)$ consists only of elements of order 2 (and 0). 
Thus it is enough to control the image of the fundamental class in

$$
H_{2}\left(\left(\mathbb{R} \mathbb{P}^{\infty}\right)^{r} ; \mathbb{Z} / 2\right) .
$$

The vanishing is equivalent to

$$
h^{*} x \cup h^{*} y=0
$$

for all $x, y \in H^{1}\left(\left(\mathbb{R} \mathbb{P}^{\infty}\right)^{r} ; \mathbb{Z} / 2\right)$. This follows since, by construction, the intersection form vanishes on $\operatorname{Ker}\left(H_{1}\left(+_{k} \mathbb{R P}^{2} ; \mathbb{Z} / 2\right) \rightarrow H_{1}\left(\left(\mathbb{R} \mathbb{P}^{\infty}\right)^{r} ; \mathbb{Z} / 2\right)\right.$, which under the isometry between $H_{1}\left(+{ }_{k} \mathbb{R} \mathbb{P}^{2} ; \mathbb{Z} / 2\right) \cong H^{1}\left(+_{k} \mathbb{R P}^{2} ; \mathbb{Z} /{ }_{2}\right)$ corresponds to the image of

$$
H^{1}\left(\left(\mathbb{R} \mathbb{P}^{\infty}\right)^{r} ; \mathbb{Z} / 2\right) \rightarrow H^{1}\left(+_{k} \mathbb{R} \mathbb{P}^{2} ; \mathbb{Z} / 2\right) .
$$

Summarizing the information so far, we have shown that

$$
\left[+{ }_{k} \mathbb{R P}^{2}, f\right]=0 \in \Omega_{2}\left(\left(\mathbb{R} \mathbb{P}^{\infty}\right)^{r} ; L\right) .
$$

Let $h: W \rightarrow\left(\mathbb{R P}^{\infty}\right)^{r}$ be a zero bordism. We claim that the kernel of the map induced by the inclusion

$$
H_{1}\left(+{ }_{k} \mathbb{R} \mathbb{P}^{2} ; \mathbb{Z} / 2\right) \rightarrow H_{1}(W ; \mathbb{Z} / 2)
$$

is our code $C$. Since $\left.h\right|_{+{ }_{k} \mathbb{R}^{2}}=f$, we conclude that

$$
\operatorname{Ker}\left(H_{1}\left(+{ }_{k} \mathbb{R P}^{2} ; \mathbb{Z} / 2\right) \rightarrow H_{1}(W ; \mathbb{Z} / 2)\right.
$$

is contained in $C$; but this kernel has dimension $r=\operatorname{dim} C$ implying the statement.

Finally we consider the classifying map $g$ of $L$ and the composition

$$
g h: W \rightarrow \mathbb{R} \mathbb{P}^{\infty},
$$

to construct the induced 2-fold covering $\hat{W}$ over $W$. Since $W$ is oriented (twisted by $L), \hat{W}$ is an orientable manifold. The boundary of $W$ is $+{ }_{k} S^{2}$ and the restriction of the deck transformation to the boundary is $-i d$ on each summand. Thus we obtain an involution $\tau$ on

$$
M:=\hat{W} \cup+{ }_{k} D^{3},
$$

which on $\hat{W}$ is the deck transformation and on each $D^{3}$ is $-i d$. By construction, the code associated to this 3-manifold $M$ and $\tau$ is the given code, finishing the argument.

The above construction depends on the choice of the zero cobordism $h: W \rightarrow\left(\mathbb{R} P^{\infty}\right)^{r}$, and it is not clear whether one obtains a manifold $M$ with involution, which has the maximal number of isolated fixed points. We will show that one can change $W$ by surgery to reduce the cohomology of $M$, and obtain a pair $(M, \tau)$ with the maximal number of isolated fixed points. By Smith theory, the maximality condition is equivalent to the injectivity of $H_{G}^{*}\left(M ; \mathbb{Z}_{2}\right) \rightarrow H_{G}^{*}\left(M^{G} ; \mathbb{Z}_{2}\right) \cong\left(\mathbb{Z}_{2}[t]\right)^{k}$, resp. the surjectivity of $H_{*}^{G}\left(M^{G} ; \mathbb{Z}_{2}\right) \rightarrow H_{*}^{G}\left(M ; \mathbb{Z}_{2}\right)$. In our case $M=\hat{W} \cup\left({ }_{k} D^{3}\right)$. The equivariant Mayer-Vietoris sequence (with $\mathbb{Z}_{2}$ coefficients) gives:

$$
\cdots \rightarrow H_{*}^{G}\left({ }_{k} S^{2}\right) \rightarrow H_{*}^{G}(\hat{W}) \oplus H_{*}^{G}\left(+_{k} D^{3}\right) \rightarrow H_{*}^{G}(M) \rightarrow H_{*-1}^{G}\left(+_{k} S^{2}\right) \rightarrow \cdots .
$$

One has $H_{*}^{G}\left(+_{k} S^{2}\right) \cong\left(H_{*}\left(+_{k} \mathbb{R} P^{2}\right)\right.$ and $H_{*}^{G}(\hat{W}) \cong H_{*}(W)$ since the actions on $+_{k} S^{2}$ and $\hat{W}$ are free, and $H_{*}^{G}\left({ }_{k} D^{3}\right) \cong H_{*}^{G}\left(M^{G}\right)$. The inclusion $S^{2} \subset D^{3}$ induces the 
inclusion $H_{*}\left(\mathbb{R} P^{2}\right) \rightarrow H_{*}\left(\mathbb{R} P^{\infty}\right)$. Hence the map $H_{*}^{G}\left(M^{G}\right) \rightarrow H_{*}^{G}(M)$ is surjective if and only if $H_{i}\left({ }_{k} \mathbb{R} P^{2}\right)=H_{i}(\partial W) \rightarrow H_{i}(W)$ is surjective for $i=1,2$. But the long exact sequence of the Poincaré pair $(W, \partial W)$ shows that surjectivity for $i=1$ already implies surjectivity for $i=2$. To verify the maximality condition it therefore suffices to show that $H_{1}(\partial W) \rightarrow H_{1}(W)$ is surjective. We want to arrive at this condition by applying surgery to $W$ (if necessary). Assume that $H_{1}(\partial W) \rightarrow H_{1}(W)$ is not surjective. We consider the following diagram:

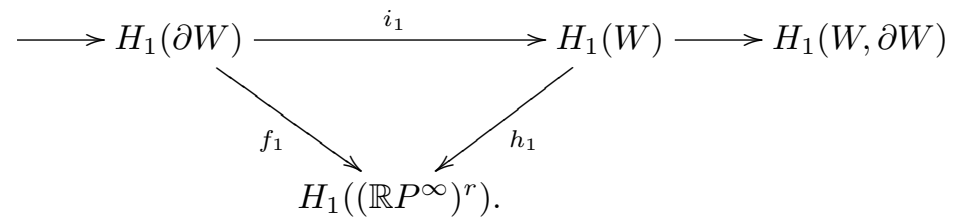

We already know that $i_{1}$ and $f_{1}$ have the same kernel, namely the code $C$, and $f_{1}$ is surjective by construction. Hence $i_{1}$ is surjective if and only if $h_{1}$ is injective. Assume that there exists an $a \in H_{1}(W), a \neq 0$, with $h_{1}(a)=0$. Then $a$ maps nontrivially to $H_{1}(W, \partial W)$ which follows since $i_{1}$ and $f_{1}$ have the same kernel. Thus by Poincaré-Lefschetz duality there is an element $b \in H_{2}(W)$ dual to $a$. Since $W$ is orientable (twisted by $L$ ), the normal bundle of an embedded circle representing $a$ is trivial. Performing surgery with respect to an embedding $S^{1} \times D^{2}$ representing $a$ kills the class $a$ and its dual $b$ with respect to the intersection pairing. This follows by an argument using the long exact homology sequence of the pair $\left(W, W \backslash S^{1} \times D^{2}\right)$ and the corresponding sequence for the manifold resulting from surgery on $S^{1} \times D^{2}$. The map $h_{1}: H_{1}(W) \rightarrow H_{1}\left(\left(\mathbb{R} P^{\infty}\right)^{r}\right)$ factors through the quotient $H_{1}(W) /\langle a\rangle$. Hence we can find a map $h^{\prime}: W^{\prime} \rightarrow\left(\mathbb{R} P^{\infty}\right)^{r}$ of our new manifold $W^{\prime}$, which restricts to $f$ on the boundary $\partial W^{\prime}=\partial W=+{ }_{k} \mathbb{R} P^{2}$. Iterating the process (if necessary) gives the following result.

Theorem 3.2. Every self-dual, binary code can be obtained from an involution on an orientable 3-manifold with the maximal number of isolated fixed points.

We say that a cohomology type of a 3-manifold admits an involution with the maximal number of fixed points, if such an involution exists on some 3-manifold with the given cohomology algebra. The above theorems then give the following corollary.

Corollary 3.3. A cohomology type of a 3-manifold admits an involution with the maximal number of isolated fixed points if and only if the cohomology algebra can be given by a self-dual, binary code.

In principle this corollary allows us to translate results about self-dual, binary codes into results about involution on a 3-manifold and vice versa. Self-dual, binary codes have been classified up to length 32 (see [2]), which is far beyond the range where there exists a reasonable classification of Poincaré duality algebras of formal dimension 3. But the code classification describes precisely those cohomology types of 3-manifolds which admit an involution with the maximal number of fixed points. As pointed out in [4], one gets as a consequence, that "most" 3-manifolds do not admit an involution with the maximal number of isolated fixed points. 


\section{Spin-structures and doubly even codes}

Doubly even codes, i.e. those for which all code words have weights zero mod 4 form a very interesting class of self-dual, binary codes. Among all self-dual, binary codes, doubly-even codes are rare, though they are very important in particular for applications (cf. [5]). It is a challenge in coding theory to find codes of moderate length, such that any non-zero code word has a rather high weight, since this would give good error-correcting properties for the code; e.g., it is a long-standing open question whether there exist a doubly even self-dual, binary code of length 72 , such that any non-zero code word has length at least 16 . We do not claim that the translation to involutions will help to answer such a question, but the relation between the two areas might exhibit interesting properties on both sides.

In view of the above corollary, the properties of codes correspond to (perhaps geometric) properties of 3-manifolds with involution. We show in this section that indeed doubly even codes correspond in a sense to Spin-structures.

Let $M$ be an oriented closed 3-manifold with involution $\tau$ having exactly $k$ isolated fixed points. From these data we will construct a 4-manifold by starting with $M \times S^{1}$ and dividing by the involution, which on $M$ is $\tau$ and on $S^{1}$ is complex conjugation. This is a manifold with $2 k$ isolated singularities, where $k$ is the number of fixed points of $\tau$. All fixed point singularities are cones over $\mathbb{R P}^{3}$, which are the links of the singularities. Since the involution on $M \times S^{1}$ is orientation-preserving, the orientation on $M \times S^{1}$ induces an orientation on the quotient (after removing the fixed points), which in turn gives an orientation on each link $\mathbb{R P}^{3}$. Now we remove open cones around the singularities and replace them by the disc bundle of the complex line bundle over $\mathbb{C P}^{1}$ with Chern class -2 . The reason for choosing this sign of the Chern class (and not +2 ) is that the induced orientation on $\mathbb{R P}^{3}$ above is the opposite of this orientation (we will discuss this in more detail in the proof of the following result). This implies that the orientations fit together and so the result is an oriented 4-manifold denoted $N(M, \tau)$. We say that $\tau$ is a Spin-involution if $N(M, \tau)$ admits a Spin-structure compatible with the given orientation.

The construction of $N(M, \tau)$ is well known in the case of the 3 -torus $T^{3}$ with $\tau$ complex conjugation. Then $N\left(T^{3}, \tau\right)$ is the $K 3$-surface, which has a Spin-structure.

Theorem 4.1. Let $M$ be a closed oriented 3-manifold with involution $\tau$ with finitely many fixed points. If $\tau$ is a Spin-involution, then the code $C(M, \tau)$ is doubly even.

Proof. We assume that the reader is familiar with $\mathrm{Pin}^{-}$-structures [3]. We recall that a $\mathrm{Pin}^{-}$-structure on a smooth manifold $M$ is a Spin-structure on $T M \oplus \operatorname{Det}(T M)$. Here we note that $T M \oplus \operatorname{Det}(T M)$ has a natural orientation, which we assume to be compatible with the Spin-structure. Thus the $\mathrm{Pin}^{-}$-structures are classified by $H^{1}(M ; \mathbb{Z} / 2)$.

A $\mathrm{Pin}^{-}$-structure on a surface $F$ determines a quadratic refinement

$$
q: H^{1}(F ; \mathbb{Z} / 2) \rightarrow \mathbb{Z} / 4,
$$

such that $q(x+y)=q(x)+q(y)+2\langle x, y\rangle$, where $\langle x, y\rangle$ is the intersection form. The two $\mathrm{Pin}^{-}$-structures on $\mathbb{R P}^{2}$ are distinguished by the quadratic form, which can take the values \pm 1 . For all this, see [3]. If $W$ is a 3 -dimensional $\mathrm{Pin}^{-}$-manifold with boundary $F$, then, on the image of $H^{1}(W ; \mathbb{Z} / 2) \rightarrow H^{1}(\partial W ; \mathbb{Z} / 2)$, the intersection form and 
the quadratic refinement vanish. This follows from [3] as explained in [6].

Now suppose that the disjoint union of $k$ copies of $\mathbb{R P}^{2}$ is the boundary of a $\mathrm{Pin}^{-}$-manifold $W$ and the induced $\mathrm{Pin}^{-}$-structure is equal on all components of the boundary. Then if $x \in H^{1}(W ; \mathbb{Z} / 2)$, and $y=i^{*}(x)=\left(y_{1}, \ldots, y_{k}\right)$, we conclude that $\sum_{i} y_{i}=0 \bmod 4$. Thus we are finished if the condition that $\tau$ is a Spin-involution implies that $\left(M \backslash+{ }_{k} B^{3}\right) / \tau$ has a $\mathrm{Pin}^{-}$-structure, which on all boundary components is the same. Here $B^{3}{ }_{i}$ is an open ball around the $i$-th fixed point.

To see this we first note that a $\mathrm{Pin}^{-}$-structure on $\mathbb{R P}^{2}$ is the same as a Spinstructure on the total space of $T \mathbb{R} \mathbb{P}^{2} \oplus \operatorname{Det} T \mathbb{R P}^{2}$. Since Det $T \mathbb{R} \mathbb{P}^{2}$ is the normal bundle of $\mathbb{R P}^{2}$ in $\mathbb{R P}^{3}$, we can, via a tubular neighbourhood, identify $T \mathbb{R P}^{2} \oplus \operatorname{Det} T \mathbb{R P}^{2}$ with an open subset of $\mathbb{R P}^{3}$, which is homotopy equivalent to $\mathbb{R P}^{3}-p t$. Thus a Pin ${ }^{-}$structure on $\mathbb{R P}^{2}$ determines a Spin-structure on $\mathbb{R P}^{3}$ and vice versa. In particular, this means that the Pin ${ }^{-}$-structure on $\mathbb{R P}^{2}$ determines an orientation on $\mathbb{R} \mathbb{P}^{3}$. We note that $\mathbb{R P}^{3}$ is the total space of the complex line bundle over $\mathbb{C P}^{1}$ with first Chern class 2. Using the complex orientation on $\mathbb{C P}^{1}$ and on the complex line bundle we obtain an orientation on $\mathbb{R P}^{3}$. It is not difficult to show that this orientation agrees with the orientation coming from the $\mathrm{Pin}^{-}$-structure on $\mathbb{R P}^{2}$ (one only has to compare the orientations at one point). Thus, if this is the orientation on a component of the boundary of some 4-manifold $V$, then we obtain an oriented manifold by gluing the disk bundle of the complex line bundle over $\mathbb{C P}^{1}$ with Chern class -2 (this induces the negative orientation compared to the orientation above, and so the orientations fit together). The key observation for our proof is that since this disc bundle is simply connected there is a unique Spin-structure on it.

Now we consider $M \times S^{1}$ with the involution given by $\tau$ and complex conjugation c. Each fixed point of $M$ and each fixed point of $S^{1}$ gives a fixed point of $M \times S^{1}$, and for each fixed point the link of the corresponding singularity in $M \times S^{1} /(\tau \times c)$ is $\mathbb{R P}^{3}$ containing the link of the corresponding singularity in $M / \tau$. Thus a $\mathrm{Pin}^{-}$structure on each link in $M / \tau$ determines a Spin-structure of the two (for each fixed point of $\left.S^{1}\right)$ corresponding links in $M \times S^{1} /(\tau \times c)$ ) and vice versa. If $N(M, \tau)$ has a Spin-structure, then this is the same on each disk bundle of the complex line bundle with Chern class -2 over $\mathbb{C P}^{1}$, since there is a unique Spin-structure with the given orientation. Thus the restriction of the $\mathrm{Pin}^{-}$-structure to each link $\mathbb{R P}^{2}$ is the same. As explained above this implies the theorem.

A by now classical result of Gleason (see e.g. [5]) says that the length of a doubly even code must be divisible by 8 .

Corollary 4.2. The total dimension of the cohomology of a 3-manifold with Spininvolution having the maximal number of isolated fixed points is divisible by 8.

Next we prove that for each doubly even self-dual code $C$, there is a 3 -manifold $M$ with Spin-involution $\tau$ such that the corresponding code is $C$.

Proposition 4.3. Let $C$ be a doubly even self-dual code. Then there is a 3-manifold $M$ with Spin-involution $\tau$ whose code is $C$.

Proof. We proceed as in the proof of Theorem 3 and use the notation from there. Now we consider $+_{k} \mathbb{R P}^{2}$ as a Pin ${ }^{-}$-manifold, where all copies are equipped with the same 
$\operatorname{Pin}^{-}$-structure, which, if we pass to the corresponding Spin-structure on $\mathbb{R P}^{3}$ can be extended to the disc bundle of the complex line bundle with first Chern class -2 over $\mathbb{C P}^{1}$. Together with the map $f$ we obtain an element of $\Omega_{2}^{\text {Pin }^{-}}\left(\mathbb{R} \mathbb{P}^{\infty}\right)^{r}$. We compute this bordism group with the Atiyah-Hirzebruch spectral sequence. From [3], we use that $\Omega_{0}^{\mathrm{Pin}^{-}}=\mathbb{Z}, \Omega_{1}^{\mathrm{Pin}^{-}}=\mathbb{Z} / 2$ and $\Omega_{2}^{\mathrm{Pin}^{-}}=\mathbb{Z} / 8$, where the latter group is generated by $\mathbb{R P}^{2}$ with any $\mathrm{Pin}^{-}$-structure.

The Atiyah-Hirzebruch spectral sequence computing $\Omega_{2}^{\text {Pin }^{-}}\left(\left(\mathbb{R} \mathbb{P}^{\infty}\right)^{r}\right)$ has the entries:

$$
\begin{gathered}
\Omega_{2}^{\mathrm{Pin}^{-}}, \\
H_{1}\left(\left(\mathbb{R} \mathbb{P}^{\infty}\right)^{r} ; \mathbb{Z} / 2\right), \\
H_{2}\left(\left(\mathbb{R} \mathbb{P}^{\infty}\right)^{r} ; \mathbb{Z}\right) .
\end{gathered}
$$

The component in the first entry is given by the $k$-fold sum of $\mathbb{R P}^{2}$ with the given $\mathrm{Pin}^{-}$-structure, which is zero if and only if $k=0 \bmod 8$. But this is the case for doubly even self-dual codes.

The last entry is as in the case of oriented bordism (twisted by $L$ ) detected by the image of the fundamental class with coefficients in $\mathbb{Z} / 2$, which, as shown before, vanishes if the code is self-dual.

The second entry is a bit delicate. We only have to detect the corresponding entry for $\Omega_{2}^{\text {Pin }^{-}}\left(\mathbb{R P}^{\infty}\right)$, since we can project to the differen components. Then the corresponding entry is in $\mathbb{Z} / 2$. By the fact that the bordism group is a module over $\Omega_{*}^{\mathrm{Pin}^{-}}$, we see that the non-trivial element is represented by $\left(S^{1} \times \eta, i p_{1}\right)$, where $\eta$ is $S^{1}$ with the non-trivial $\mathrm{Pin}^{-}$-structure (which for 1-manifolds is the same as a Spinstructure) and $i$ is the inclusion $S^{1} \rightarrow \mathbb{R P}^{\infty}$. We are free to choose a $\mathrm{Pin}^{-}$-structure on the first factor. If we choose the Spin-structure again to be the non-trivial one, we see that the induced 2-fold cover is $\eta \times \eta$, which is the non-trivial element in $\Omega_{2}^{\text {Spin }}$. We note that whatever $\mathrm{Pin}^{-}$-structure we choose on the first factor, we can change it, if necessary, to the non-trivial one, by modifying it with the non-trivial element in the image of $H^{1}\left(\mathbb{R} P^{\infty} ; \mathbb{Z} / 2\right)$. The upshot of these considerations is that we can detect the second term in the Atiyah-Hirzebruch spectral sequence of an element $[N, g] \in \Omega_{2}^{\text {Pin }^{-}}\left(\mathbb{R P}^{\infty}\right)$, whose underlying Pin ${ }^{-}$-bordism class is zero and whose fundamental class maps to zero by the following criterion: It is zero if and only if, for all modifications of the $\mathrm{Pin}^{-}$-structure by elements in $H^{1}\left(\mathbb{R P}^{\infty} ; \mathbb{Z} / 2\right)$, the induced 2 -fold covering is zero bordant. Applying this to the case where $N={ }_{8 l} \mathbb{R P}^{2}$, we note that the induced covering is an $S^{2}$ over each summand which maps non-trivially to $\mathbb{R} \mathbb{P}^{\infty}$ (which is zero bordant), and that it is $\mathbb{R} \mathbb{P}^{2}+\mathbb{R P}^{2}$ for each summand which maps trivially. But since $\mathbb{R P}^{2}$ is a generator of $\Omega_{2}^{\mathrm{Pin}^{-}} \cong \mathbb{Z} / 8$, this implies that if the number of summands, which are mapped trivially, is $0 \bmod 4$, then the bordism class is trivial. Returning to the situation given by our code we see that if the code is doubly even, then this criterion applies.

Thus we have shown that for doubly even codes the bordism class vanishes in $\Omega_{2}\left(\left(\mathbb{R} \mathbb{P}^{\infty}\right)^{r}\right)$, and as in the proof of Theorem 2 we construct a 3-manifold $M$ with involution $\tau$ giving the code. Since the $\mathrm{Pin}^{-}$-structure is the same for all copies of $\mathbb{R P}^{2}$, we obtain a Spin-involution. Namely, the 4-manifold we construct is the blow up of a Spin-manifold obtained by replacing the open cones over the individual $\mathbb{R P}^{3}$ 's 
by the disc bundle of the complex line bundle with Chern class -2 over $\mathbb{C P}^{1}$. After perhaps changing the orientation before the gluing, the resulting manifold is oriented. Since the Spin-structure on all $\mathbb{R P}^{3}$ 's extend to this disc bundle, the manifold is a Spin-manifold.

As before one can apply surgery, this time taking into account the $\mathrm{Pin}^{-}$-structure to get the following result.

Theorem 4.4. Every binary, doubly even, self-dual code can be obtained from a Spin-involution with the maximal number of isolated fixed points on an orientable 3-manifold.

\section{Acknowledgements}

The first author was partially supported by a DFG grant. He also would like to thank the Mathematics Department of Columbia University and the Courant Institute for support and stimulating atmosphere while parts of the project were carried out.

\section{References}

[1] C. Allday and V. Puppe, Cohomological methods in transformation groups, Cambridge Studies in Advanced Mathematics, vol. 32, Cambridge University Press, Cambridge, 1993. MR 1236839 (94g:55009).

[2] J.H. Conway, V. Pless, and N.J.A. Sloane, The binary self-dual codes of length up to 32: a revised enumeration, J. Combin. Theory Ser. A 60 (1992), no. 2, 183-195. MR 1168153 (93e:11150).

[3] R.C. Kirby and L.R. Taylor, Pin structures on low-dimensional manifolds, Geometry of low-dimensional manifolds, 2 (Durham, 1989), London Math. Soc. Lecture Note Ser., vol. 151, Cambridge Univ. Press, Cambridge, 1990, pp. 177-242. MR 1171915 (94b:57031).

[4] V. Puppe, Group actions and codes, Canad. J. Math. 53 (2001), no. 1, 212-224. MR 1814971 (2001m:55010).

[5] Eric M. Rains and N.J.A. Sloane, Self-dual codes, Handbook of coding theory, Vol. I, II, North-Holland, Amsterdam, 1998, pp. 177-294. MR 1667939.

[6] L.R. Taylor, Quadratic enhancements of surfaces: two vanishing results (2008), available at arXiv:0802.0111.

Matthias Kreck kreck@him.uni-bonn.de

Hausdorff Research Institute for Mathematics, Poppelsdorfer Allee 45, 53115 Bonn, Germany

Volker Puppe Volker.Puppe@uni-konstanz.de

Universität Konstanz, Fachbereich für Mathematik und Statistik, 78457 Konstanz Germany 